\title{
De-correlation stretch filtering approach for effective Poisson reduction in galaxy Images
}

\author{
A.Sathesh \\ Lecturer, EEE Department, Eritrea Institute of Technology, Eritrea, Northeast Africa \\ sathesh4you@gmail.com \\ J. Samuel Manoharan \\ Professor \& Head, ECE Department, Bharathiyar College of Engineering, Karaikal, \\ South India \\ samuel1530@gmail.com
}

\section{ABSTRACT}

Noise reduction is one of the most important processes to enhance the quality of images. This paper proposes a statistical filter, the decorrelation stretch filter for the reduction of Poisson noise that occurs frequently in galaxy images. The primary purpose of decorrelation stretch is visual enhancement. Decorrstretch is applied to the three band images but can also work on arbitrary number of bands. This filter enhances the color separation of an image with significant band-band correlation. Effectiveness of the proposed filter is compared on the basis of Peak Signal to Noise Ratio (PSNR), Mean Square Error (MSE).

\section{Indexing terms/Keywords}

Poisson noise, Galaxy images, spatial filters, Decorrelation stretch.

\section{Academic Discipline And Sub-Disciplines}

Digital Image processing, Image denoising, Galaxy image handling

\section{SUBJECT CLASSIFICATION}

Digital galaxy images analysis with Linear algebra

\section{TYPE (METHOD/APPROACH)}

Simulation analysis

\section{Council for Innovative Research}

Peer Review Research Publishing System

Journal: INTERNATIONAL JOURNAL OF COMPUTERS \& TECHNOLOGY

Vol 11, No. 9 


\section{INTRODUCTION}

Galaxies are space systems composed of dust, gas and countless stars. They are classified into three types spiral galaxies, elliptical galaxies and irregular galaxies. Here galaxy images are considered so that any new object appearing in the galaxy can be identified. The major noise occurring in galaxy is the poisson noise.

A galaxy is a massive, gravitationally bound system that consists of stars and stellar remnants, an interstellar medium of gas dust, and an important but poorly understood component tentatively dubbed dark matter.Typical galaxies range from dwarfs with as few as ten million $\left(10^{*}\right)$ stars, up to giants with a hundred trillion $\left(10^{14}\right)$ stars, all orbiting the galaxy's center of mass. Galaxies may contain many star systems, star clusters, and various interstellar clouds.

Historically, galaxies have been categorized according to their apparent shape (usually referred to as their visual morphology). A common form is the elliptical galaxy, which has an ellipse-shaped light profile. Spiral galaxies are diskshaped assemblages with dusty, curving arms. Galaxies with irregular or unusual shapes are known as irregular galaxies, and typically result from disruption by the gravitational pull of neighboring galaxies. Such interactions between nearby galaxies, which may ultimately result in galaxies merging, may induce episodes of significantly increased star formation, producing what is called a starburst galaxy. Small galaxies that lack a coherent structure could also be referred to as irregular galaxies.

There are probably more than 170 billion $\left(1.7 \times 10^{11}\right)$ galaxies in the observable universe. Most galaxies are 1,000 to 100,000 parsecs in diameter and are usually separated by distances on the order of millions of parsecs (or megaparsecs). Intergalactic space (the space between galaxies) is filled with a tenuous gas of an average density less than one atom per cubic meter. The majority of galaxies are organized into a hierarchy of associations called clusters, which, in turn, can form larger groups called superclusters. These larger structures are generally arranged into sheets and filaments, which surround immense voids in the universe.

Although it is not yet well understood, dark matter appears to account for around $90 \%$ of the mass of most galaxies. Observational data suggests that super massive black holes may exist at the center of many, if not all, galaxies. They are proposed to be the primary cause of active galactic nuclei found at the core of some galaxies. The Milky Way galaxy appears to harbor at least one such object within its nucleus.

Poisson noise is a dominant noise that occurs in the lighter parts of the image due to quantum fluctuations. This noise is also called as shot noise. It has a root mean square value proportional to the square root of image intensity. The noise at different pixels is independent of one another. This Poisson noise can be reduced by using some spatial filters. In this paper, a statistical filter- decorrelation stretch is proposed for Poisson reduction considering the multiplicative characteristics of Poisson.

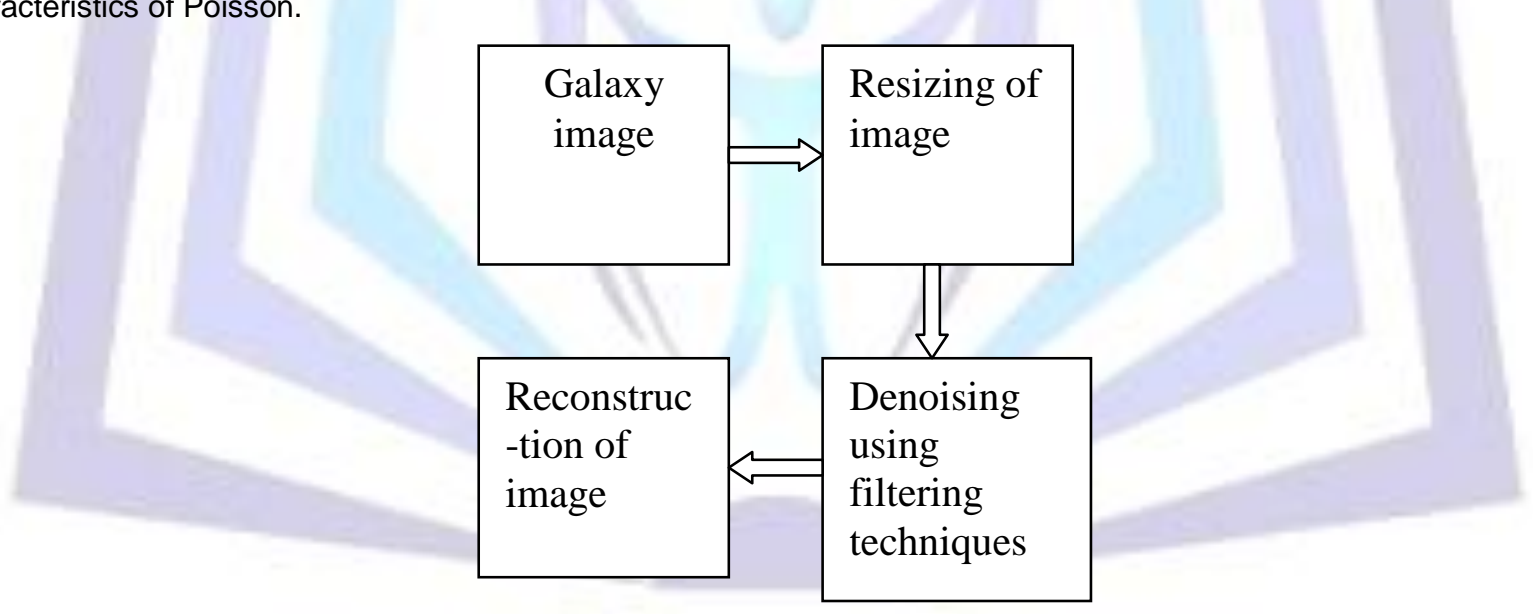

Fig.1. Overview of this paper

A galaxy image is taken and resized to the required dimension and denoised using a variety of spatial filters. The image is then reconstructed.

The first section deals with the spatial filtering techniques. The filters discussed are median, image adjust, adaptive histogram equalization, stretch limit, min and max filter. The second section explains about our proposed technique using the decorrelation stretch filter. In the final section the outputs of different filters are compared. 


\section{INPUT IMAGES}

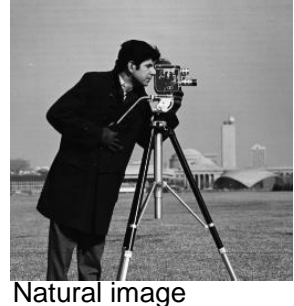

Natural image

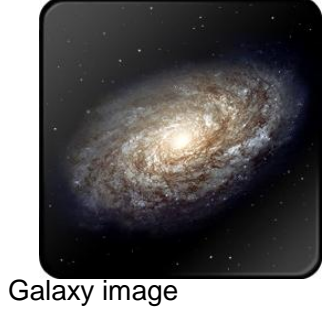

Galaxy image

\section{SPATIAL FILTERING TECHNIQUES}

\section{Median Filter}

The median filter is a nonlinear digital filtering technique used to remove noise. Median filtering is widely used in digital image processing because under certain conditions, it preserves edges while removing noise. The main idea of the median filter is to run through the signal entry by entry, replacing each entry with the median of neighboring entries. The pattern of neighbors is called the "window", which slides, entry by entry, over the entire signal. Median filtering is a kind of smoothing technique used to preserve the edges. Edges are of critical importance to the visual appearance of images.

\section{Image adjust Filter}

Imadjust maps the values in intensity image to new values such that values between low in and high in map to values between low out and high out. Values below low in and above high in are clipped; that is, values below low in map to low out, and those above high in map to high out. Imadjust transforms the colormap associated with an indexed image. If low in, high in, low out, high out, and gamma are scalars, then the same mapping applies to red, green and blue components.

\section{Adaptive histogram equalization}

Adapthisteq is used to adjust the contrast in an intensity image. The original image has low contrast, with most values in the middle of the intensity range. Adapthisteq operates on small regions in the image, called tiles. Each tile's contrast is enhanced, so that the histogram of the output region approximately matches a specified histogram. After performing the equalization, adapthisteq combines neighboring tiles using bilinear interpolation to eliminate artificially induced boundaries. To avoid amplifying any noise that might be present in the image adapthisteq can be used. Adapthisteq is used to adjust the contrast in an intensity image. The original image has low contrast, with most values in the middle of the intensity range. Adapthisteq produces an output image having values evenly distributed throughout the range.

\section{Stretch limit filter}

Stretchlim returns a pair of intensities that can be used by imadjust to increase the contrast of an image. TOL $=$ [LOW_FRACT HIGH_FRACT] specifies the fraction of the image to saturate at low and high intensities. If TOL is a scalar, TOL $=$ LOW_FRACT, and HIGH_FRACT $=1-$ LOW_FRACT, which saturates equal fractions at low and high intensities. If the argument is omitted, TOL defaults to $[0.010 .99]$, saturating $2 \%$. If $\mathrm{TOL}=0, \mathrm{LOW} \mathrm{HIGH}=[\min (\mathrm{I}(:)) \max (\mathrm{I}(:))]$. LOW_HIGH = stretchlim(RGB,TOL) returns a 2-by-3 matrix of intensity pairs to saturate each plane of the RGB image. TOL specifies the same fractions of saturation for each plane.

\section{PROPOSED TECHNIQUE}

\section{De-correlation Stretching}

De-correlation stretching enhances the color separation of an image with significant band-band correlation. The exaggerated colors improve visual interpretation and make feature discrimination easier. The number of color bands, NBANDS, in the image is usually three. But you can apply de-correlation stretching regardless of the number of color bands. The original color values of the image are mapped to a new set of color values with a wider range. The color intensities of each pixel are transformed into the color Eigen space of the NBANDS-by-NBANDS covariance or correlation matrix, stretched to equalize the band variances, and then transformed back to the original color bands.

\section{EXPERIMENTAL RESULT}

In this paper, image of size $256 \times 256$ has been used. The simulation has been done with the help of Matlab 7.6 software. Peak Signal to Noise Ratio (PSNR) is computed using Equation 2. The PSNR is higher for a better transformed image. The Mean Square Error (MSE) and the Peak Signal to Noise Ratio (PSNR) are the two error metrics used to compare image compression quality. The MSE represents the cumulative squared error between the compressed and the original image, whereas PSNR represents a measure of the peak error. The lower the value of MSE, lower is the error. 


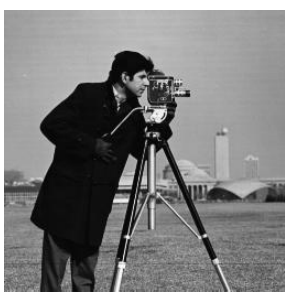

Original

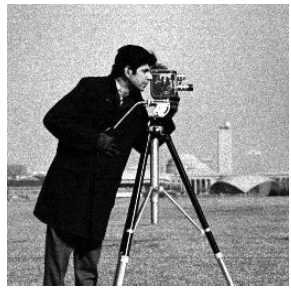

[a]

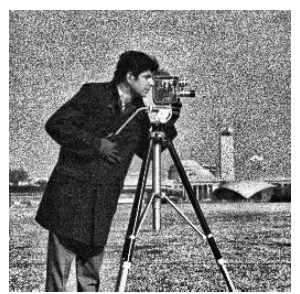

Noised

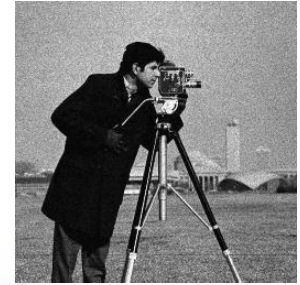

[b]

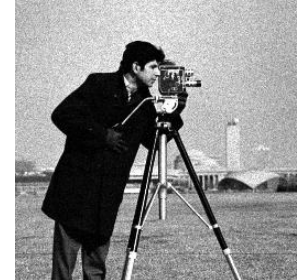

$[c]$

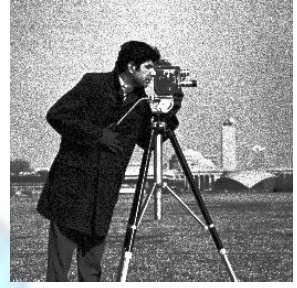

[e]

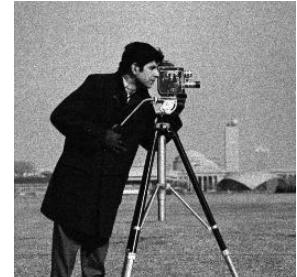

[d]

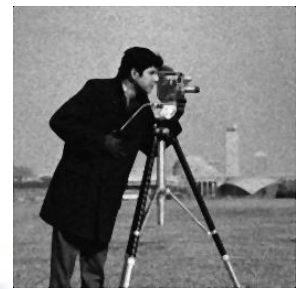

[f]

Fig. 2 Denoising of natural images using different filters [a] imadjust filtered output

[b] decorrelation stretch filtered output [c] contrast stretch image

[d] adaptive histogram equalisation filtered output [e] histogram equalisation filtered output [f] median filtered output.

$$
\begin{aligned}
& M S E=\sum\left[11(m, n)-12(m, n)^{\wedge} 2\right] / m x n \ldots . .(1) \\
& P S N R=10^{\star} \log 10\left(\left(255^{\wedge} 2\right) / m s e\right) \ldots \ldots . .(2)
\end{aligned}
$$

Denoising of images using decorrelation stretch filter has yielded best results. The PSNR value is very high for both natural and galaxy images when they are filtered using decorrelation stretch filter. Hence this approach can be used for effective denoising.

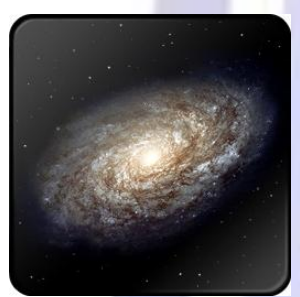

Original

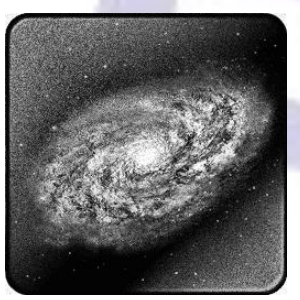

[a]

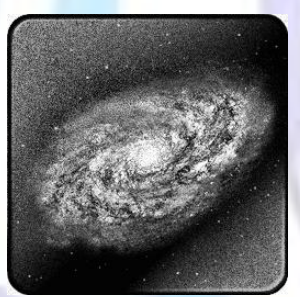

Noised

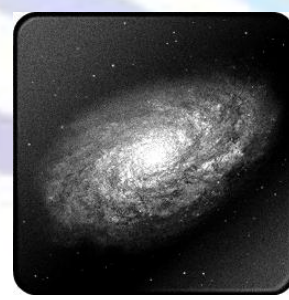

[b]

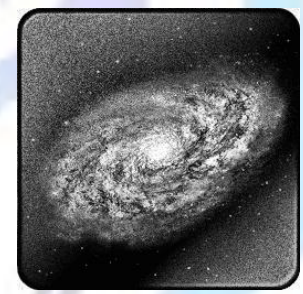

[c]

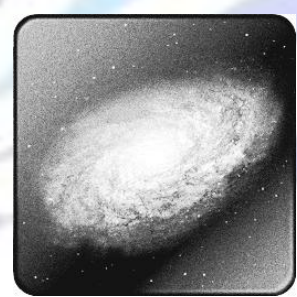

[e]

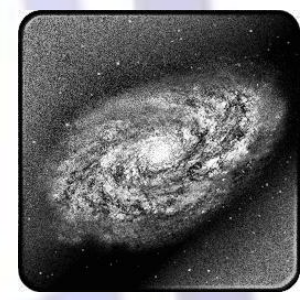

[d]

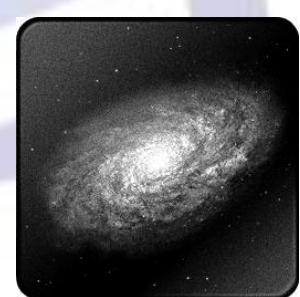

[f]

Fig. 3 Denoising of galaxy image using different filters [a] imadjust filtered output

[b] decorrelation stretch filtered output [c] contrast stretch image [e] histogram equalisation filtered output

[d] adaptive histogram equalisation filtered output [f] median filtered output 


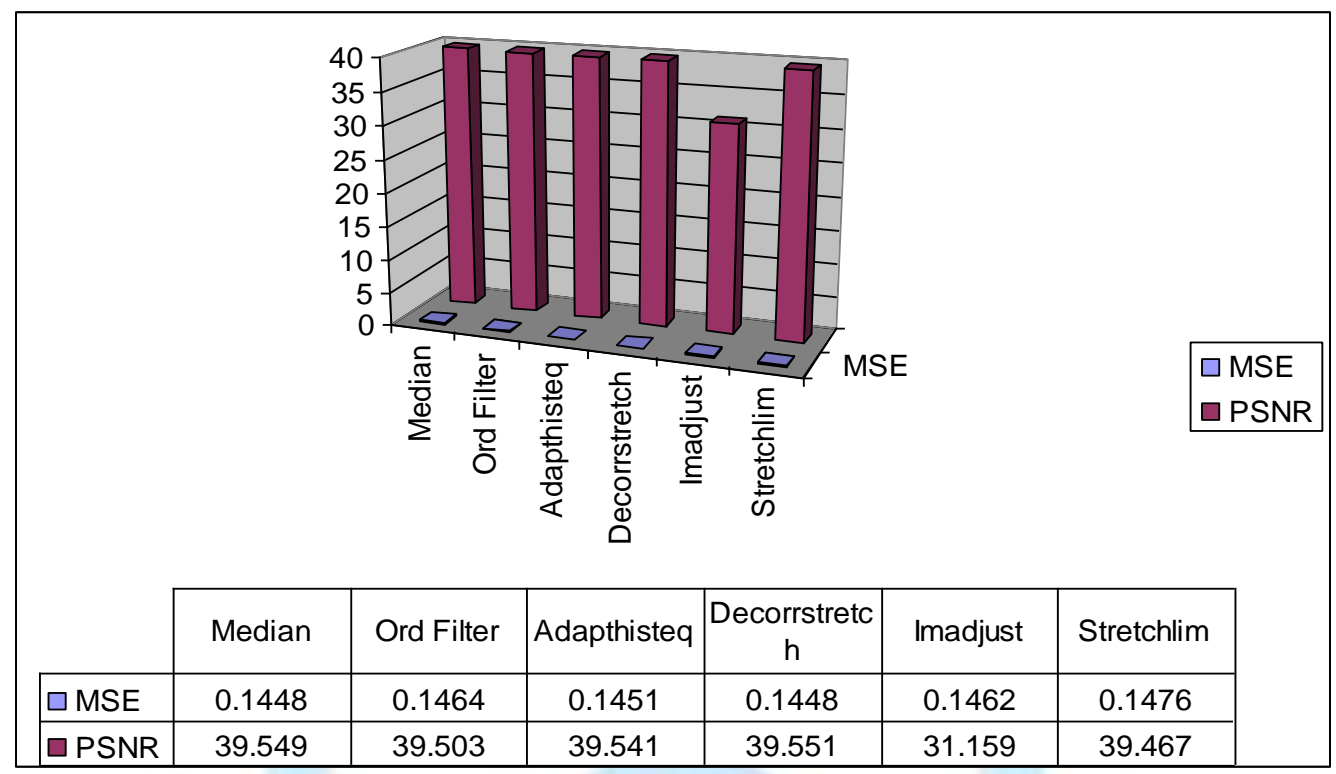

Fig $4(a)$

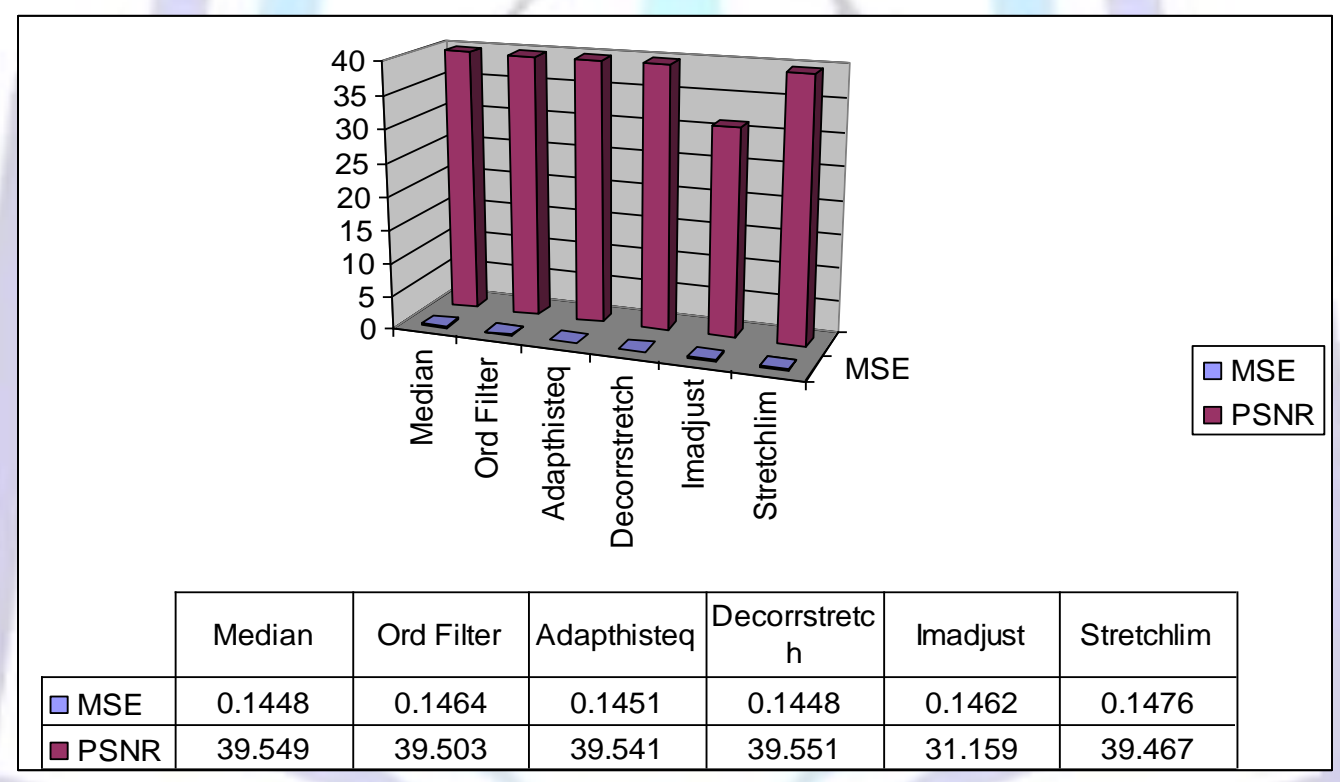

Fig 4(b)

Fig. 4.a Performance analysis graph for natural image

Fig. 4.b Performance analysis graph for galaxy image

The above graph shows the performance analysis of the different spatial filters and the PSNR and MSE values. From the graph it can be concluded that the PSNR value is maximum for the decorrelation stretch filter. 


\section{TABLE 1 COMPARISON OF OUTPUTS OBTAINED FROM DIFFERENT FILTERS}

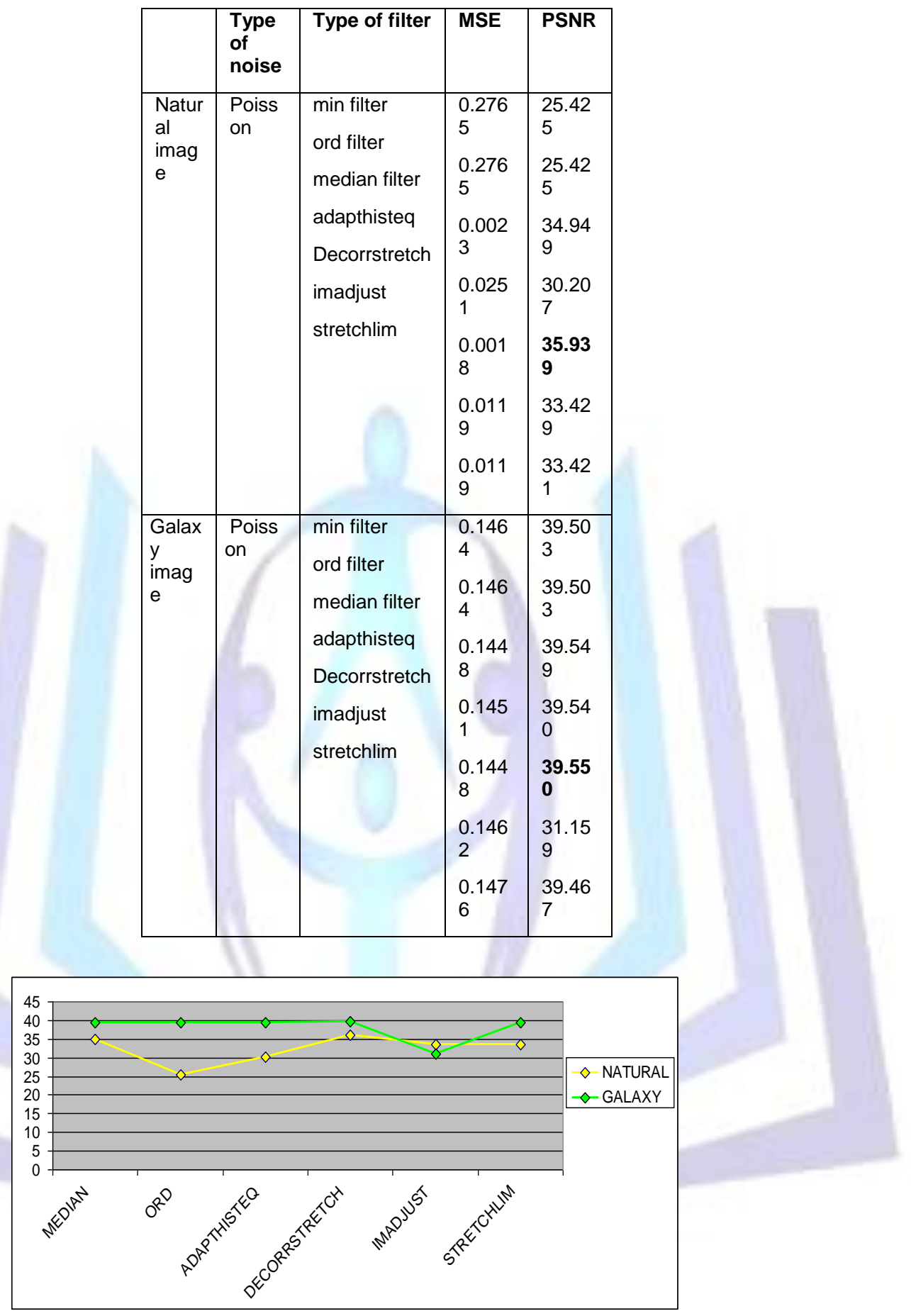

Fig. 5 Graph comparing the spatial filters for natural and galaxy images

\section{CONCLUSION}

Denoising is carried out for natural and galaxy images with Poisson noise using the statistical filters and proposed de-correlation filter. The above figures show the original image and its noisy version. Simulations are carried out in MATLAB. The performances of different denoising schemes are compared in Table1. The enhancement of images has been carried out in spatial domain. From the results obtained for both natural and galaxy images it has been found that decorrelation stretch provides better results among spatial filters.

\section{ACKNOWLEDGEMENT}

We would like to thank the anonymous reviewers whose comments have greatly improved the paper. 


\section{REFERENCES}

[1] Christos P. Loizou, Constantinos S. Pattichis, Speckle Filtering Algorithms and Software and Software for Ultrasound Imaging, Morgan and Claypool Publishers, 2008.

[2] E.R.Davies, Machine vision Theory, Algorithms, Practicalities, Elsevier, 2006.

[3] R.Gonzalez and R.Woods, Digital image processing, 2nd edition, prentice hall, 2002.

[4] Z.Wang, A.Bovik, H,Sheik and E.Simoncelli, "Image Quality assessment: From error measurement to structural similarity, "IEEE Transaction on image processing,Vol.13,No.4,pp.600-612,April,2004.

[5]Z.Wang, A.Bovik, "A Universal Quality index," IEEE Signal Processing Letters, vol.9, no.3, pp.81- 84, March, 2002.

[6] Han, X. H., Chen, Y. W. and Nakao, Z. (2003) An ICA-based method for Poisson noise reduction. Lecture Note in Artificial Intelligence, pp. 1449-1454. Springer, Berlin

[7] Karbelkar, S. N. (2005) Algorithm for calculating Poisson noise on image intensity correlations. Journal of the Optical Society of America, 7:7, pp. 1332-1336.

[8] Lasota, S. and Niemiro, W. (2003) A version of the Swendsen-Wang algorithm for restoration of images degraded by Poisson noise. Pattern Recognition, 36, pp. 931-941.

[9] Chan, T. F. and Chen, K. (2006) An optimization-based multilevel algorithm for total variation image denoising. SIAM Journal of MultiscaleModeling and Simulations, 5, pp. 615-645.

[10] Chan, T. F. and Chen, K. (2006) On a nonlinear multigrid algorithm with primal relaxation for the image total variation minimisation. Numerical Algorithms, 41, pp. 387-411.

[11] Kervrann, C. and Trubuil, A. (2004) An adaptive window approach for Poisson noise reduction and structure preserving in confocal microscopy. Paper presented at the International Symposium on Biomedical Imaging (ISBI'04, Arlington, VA.

\section{Author' biography with Photo}

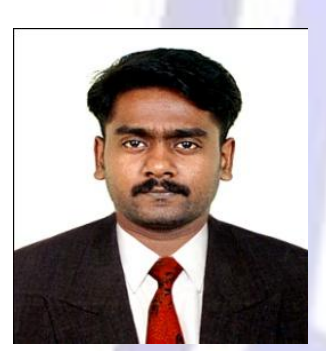

Sathesh completed his Masters in the year 2007 and has published a number of papers in national and international journals. His areas of interests include wavelets and multi resolution transforms for image denoising. Currently he is occupying a academic position in Eritrea after having worked in a reputed University in South India for the past 4 years. He is pursuing his research work in the area of complex wavelets for image approximations.

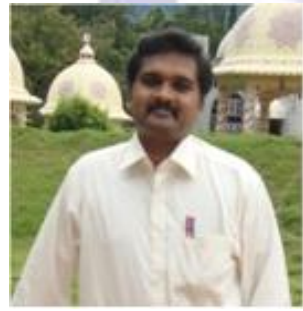

J. Samuel Manoharan obtained his Masters in 2006 and his doctorate in 2013 in the area of digital image processing. His research area includes multi resolution transforms for approximation of image components and cryptography. His interests are focussed towards processing of bio medical images. He has published several papers in reputed journals and conferences. 